\title{
IoT based Clinical Sensor Data Management and Transfer using Blockchain Technology
}

\author{
Dr. Haoxiang Wang, \\ Department of Electrical and Computer Engineering, \\ Cornell University, Ithaca, USA. \\ Email: wanghaoxiang1102@hotmail.com
}

\begin{abstract}
There has been revolutionary developments in the healthcare industry with the advancement of technology over the past years. Internet of Things, Cloud Computing, Blockchain technology, lab-on-chip, non-invasive and minimally invasive surgeries and so on has simplified several dreadful diseases. The research as well as healthcare industry have been greatly impacted by these new technologies. Clinical exams and self-health tracking can be done by means of miniaturized healthcare sensors that are powered by IoT. They help in early diagnosis and treatment guidance by clinicians at remote locations without directly being in contact with the users. The access control structures and inconsistent security policies have been a hinderance in meeting the security requirements of these data. Blockchain based smart contracts and enterprise-distributed ledger framework can be used for monitoring the vital signs of the patient. This enables accessing medical information of patients globally at any time along with immutable and extensive history log. In comparison with the traditional patient monitoring system, the proposed system offers better monitoring, improved connectivity and enhanced data security.
\end{abstract}

Keywords: Internet of Things; Cloud Storage; Blockchain technology; Patient monitoring; Data Security;

\section{Introduction}

Various health facility providers such as pharmacies, healthcare and hospitals are equipped with a diverse range of resources [1]. The physical devices are connected largely with wearable sensors by tremendous growth Information and Communication Technology (ICT). The electronic devices and software used for this purpose provides efficient large-scale data generation, processing and collection. Fatal diseases like pneumonia, influenza, cancer, heart diseases and so on have significantly increased despite the numerous health facilities available [2]. With technological revolution, sensing devices that can collect, process, and analyse patient information has been employed for continuous monitoring and observation of the vital signs. This data is shared to hospitals and other relevant departments for providing healthcare services. Legal interoperability issues are a matter of concern in this scenario. Centralization of the medical data management and sharing system will improve the efficiency of delivering healthcare services [3].

During pandemic conditions such as Covid-19, in order to reduce the risk of infection, patient movement has to be reduced [4]. For this purpose, tele-healthcare service is of great use. The medical centers and hospitals have only been partially using IoT and cloud computing technologies. However, complete implementation of these technologies with blockchain based security is essential for complete remote healthcare services [5]. The data exchange privacy, security and integrity are improved with the wide adoption of ICT paradigms. It is crucial that the clinical data that is shared must not be corrupted and have to be certified for prevention of illegal, accidental or intentional data manipulation. The privacy of the patient must also be guaranteed [6]. 
Journal of ISMAC (2020)

Vol.02/ No.03

Pages: $154-159$

http://irojournals.com/iroismac/

DOI: https://doi.org/10.36548/jismac.2020.3.003

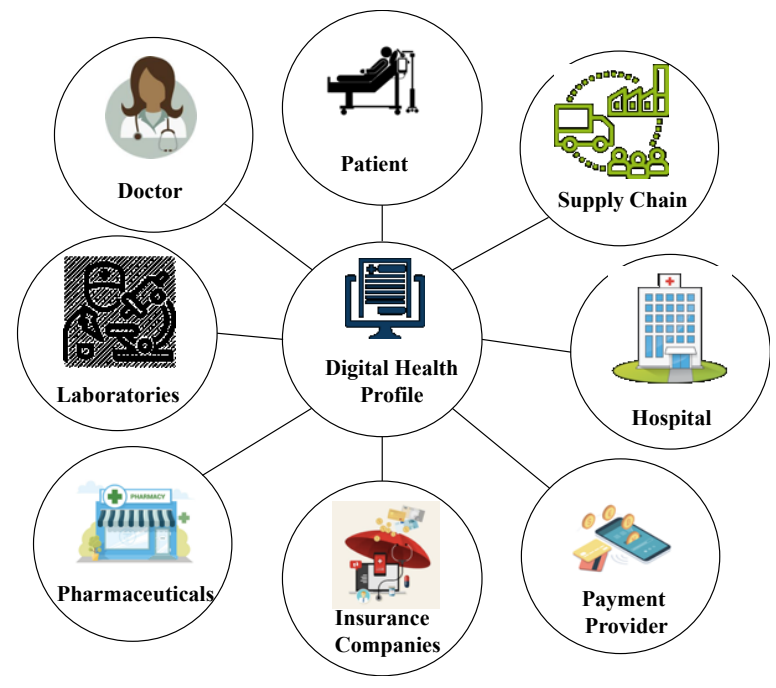

Figure 1: Blockchain technology based Healthcare applications

This paper proposes the integration of IoT, Blockchain and Cloud technologies in the medical environment for offering healthcare and tele-medical laboratory services. It allows monitoring the vital signs of the patient in a secure manner in smart hospital or remote environment [7]. Data sharing is authorized by blockchain technology. Figure 1 provides the various healthcare applications associated with blockchain technology. A web based frontend technology is developed using JavaScript and HTML for this purpose which is used for user interface [8]. Representational State Transfer Application Programming Interfaces (REST API) is used for providing product-centric services using blockchain network. Only authorized system users can control the IoT devices connected to the healthcare sensory module.

\section{Related Works}

Several researchers have tested the implementation of blockchain technology in various domains inclusive of healthcare. The hospital information systems security can be improved drastically on application of blockchain [9]. The early stage and theoretical scientific initiatives do not provide clear information regarding the framework and protocols required for implementing this technology in real time healthcare environment. Open information access concerns are addressed by blockchain on a larger scale [10]. Greater efficiency, traceability, privacy, security and transparency can be achieved on implementing this technology in healthcare services. Authorized access can be provided using blockchain for the medical information of patients. This helps in preserving the identity and integrity of the clinical history of the patient [11].

Pharmaceuticals, biomedical, neuroscience, genomics medicine, electronic health records (EHR), clinical facts, workflows, decision-making and such purposes are served in healthcare applications [12]. Efficient healthcare services can be provided using communication protocols and data standardization using IoT technologies. Data interoperability reduction, patient data security, user interfaces and better connectivity are the benefits of efficient healthcare services. A great focus is laid on the healthcare industry and towards developing reliable healthcare applications by several researchers [13]. It is essential to improve the security in accessing 
Journal of ISMAC (2020)

Vol.02/ No.03

Pages: $154-159$

http://irojournals.com/iroismac/

DOI: https://doi.org/10.36548/jismac.2020.3.003

healthcare records by stakeholders like pharmacies, hospitals and patients without any data manipulation. These challenges can be addressed by blockchain technology [14].

\section{Proposed Work}
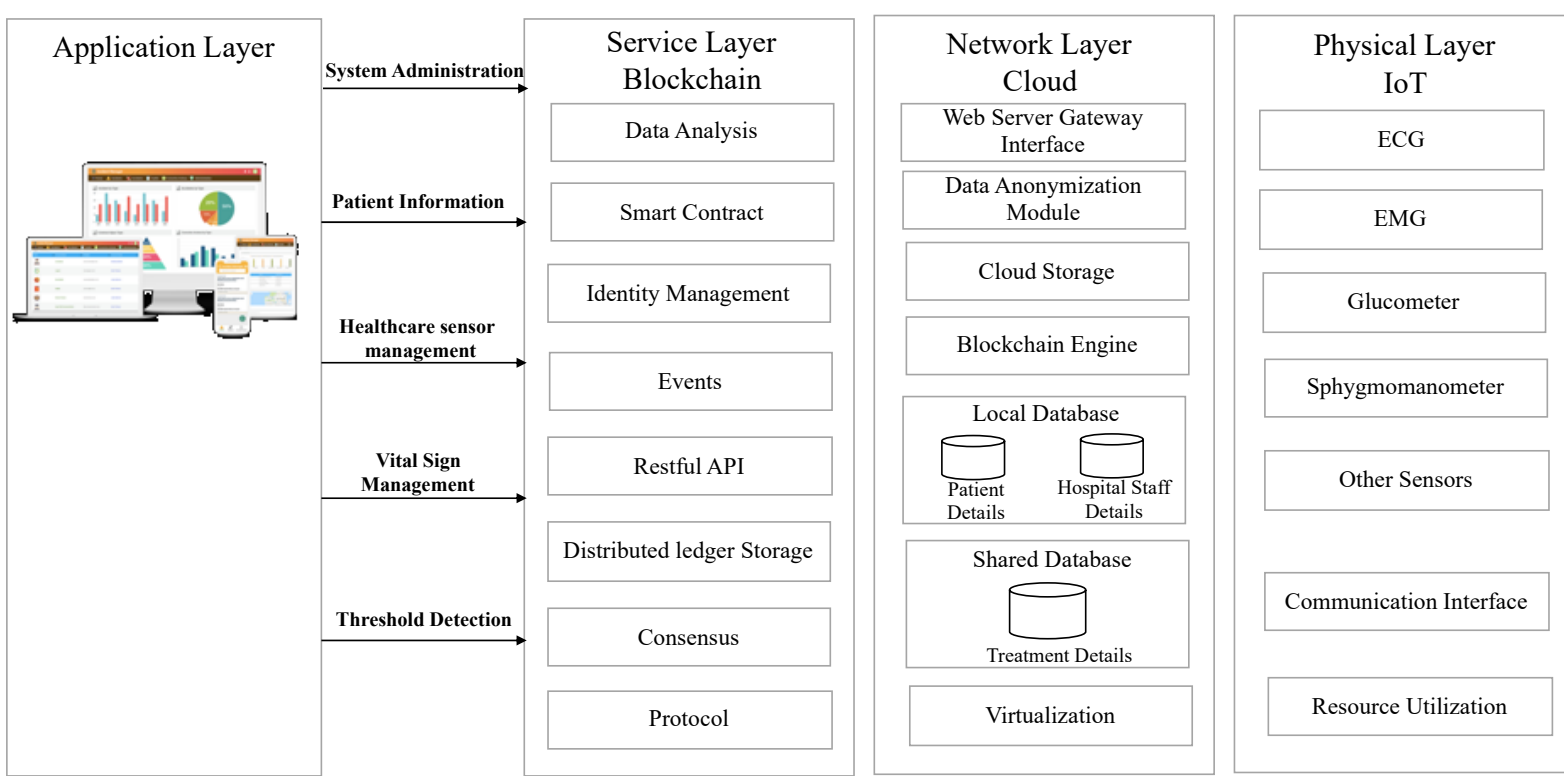

Figure 2: IoT, Cloud and Blockchain based Federated Layered healthcare system Architecture

Figure 2 provides the architecture of the proposed healthcare system in which each layer is an integration of different technologies. Developers and modify the system by addition or deletion of modules using the decoupled feature without affecting the other modules or the system on whole. The proposed model comprises of four layers namely application layer, blockchain based service layer, cloud based network layer and IoT based physical layer [15]. The IoT layer comprises of communication, data storage as well as computational healthcare devices. The cloud based network layer provides connectivity, storage, blockchain engine as well as virtualization features. The blockchain based service layer provides peer-to-peer communication (P2P), distributed ledger technology (DLT), identity management, consensus and other related services.

Digital data that is replicated, synchronized and shared are contained in the DLT. This information is distributed across the blockchain network and a ledger copy is available with every participant. The healthcare sensor information is also stored and secured by the DLT. Any modifications in this information is reflected across the blockchain in all copies making it more reliable and efficient. Smart contract code helps in modifying, accessing and managing the ledger via external client applications. The information regarding addition of new blocks in the ledger is notified through event management. The blockchain platform is accessed through certain applications and the blockchain network is managed by several services in the application programming interface (API). The assets and resources of the user are shared securely and communicated using blockchain technology. Asymmetric ciphers, consensus algorithms and P2P networks are used for blockchain based communication. Healthcare devices can be managed and controlled as well as visualization of vital signs is made possible with the user interface of the application layer as represented in Figure 2. 
Journal of ISMAC (2020)

Vol.02/ No.03

Pages: $154-159$

http://irojournals.com/iroismac/

DOI: https://doi.org/10.36548/jismac.2020.3.003

Irreversible and trackable actions are performed in the virtual healthcare system. Smart contract coding and development offers a distribution certificate that is decentralized. Laboratory tests, Computed Tomography (CT) scans, blood test and such tests are analyzed disease identification. Authorization and sharing of these files are done by open architecture of blockchain technology. Cloud storage makes use of NoSQL database for storing the patient information database and blockchain transaction hashes help in efficient and fast data search that is linked to the cloud storage files. Flexibility is provided to the user to perform operations with the healthcare sensors such as using nasal airflow sensor for reading rate of airflow or ECG sensor for reading heart rate. Body temperature sensor, EMG sensor, sphygmomanometer sensor, ECG sensor and other sensor based on the patient requirements can be included in the system. The business network domain model and roles of each participant in it can be defined by authorization and authentication of the participants through the Access Control Language (ACL)

\section{Results and Discussion}

The time-to-mine comparison of two approaches is expressed in seconds in Figure 3 for the Ethereum network approach analysis on public and hybrid domains based on the change in treatment registration requests received. The treatment registration request count is represented in the $\mathrm{x}$-axis and processing time is represented in the y-axis. It can be observed from the graph that the treatment registration requests trend is similar for both approaches for up to around 100 values after which it increases. On comparison, it is found that better performance is offered by hybrid Ethereum network by reducing the mining queue waiting time.

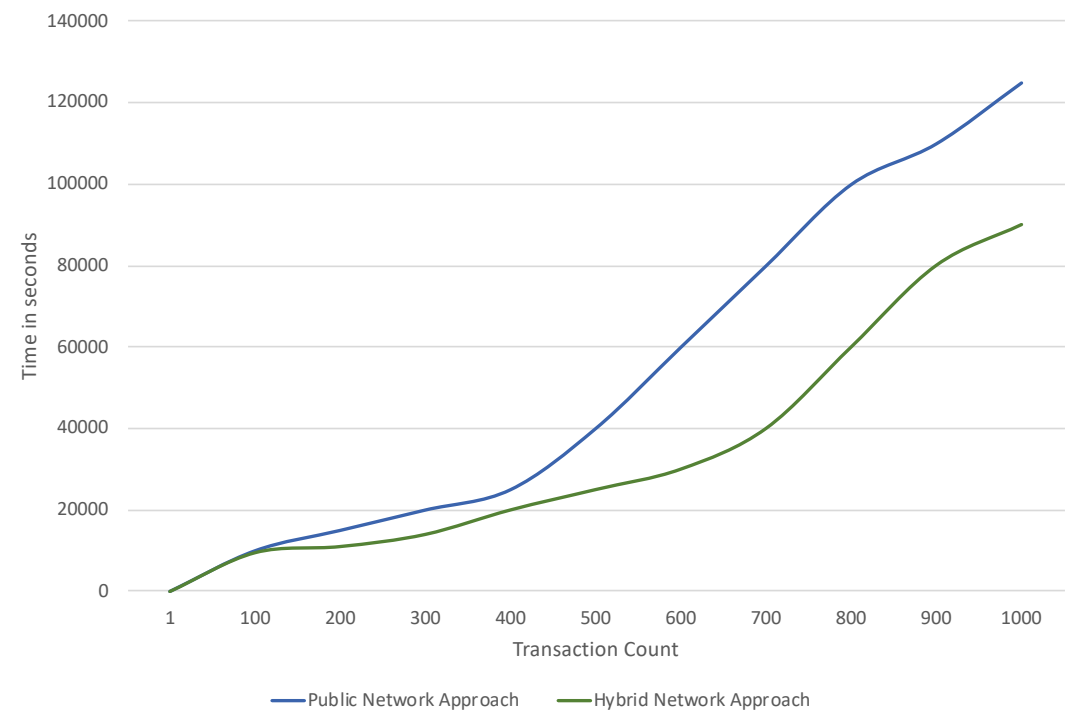

Figure 3: Ethereum network approach analysis for time vs treatment registration

Maximum, minimum and average latency for three user groups for the proposed system the invoke transaction execution latency is investigated as represented in figure 4. Users are categorized into groups as multiples of 100 from 1 to 1000 . There is a negligible difference in the increase of average latency with the increase in the number of users. 
Journal of ISMAC (2020)

Vol.02/ No.03

Pages: $154-159$

http://irojournals.com/iroismac/

DOI: https://doi.org/10.36548/jismac.2020.3.003

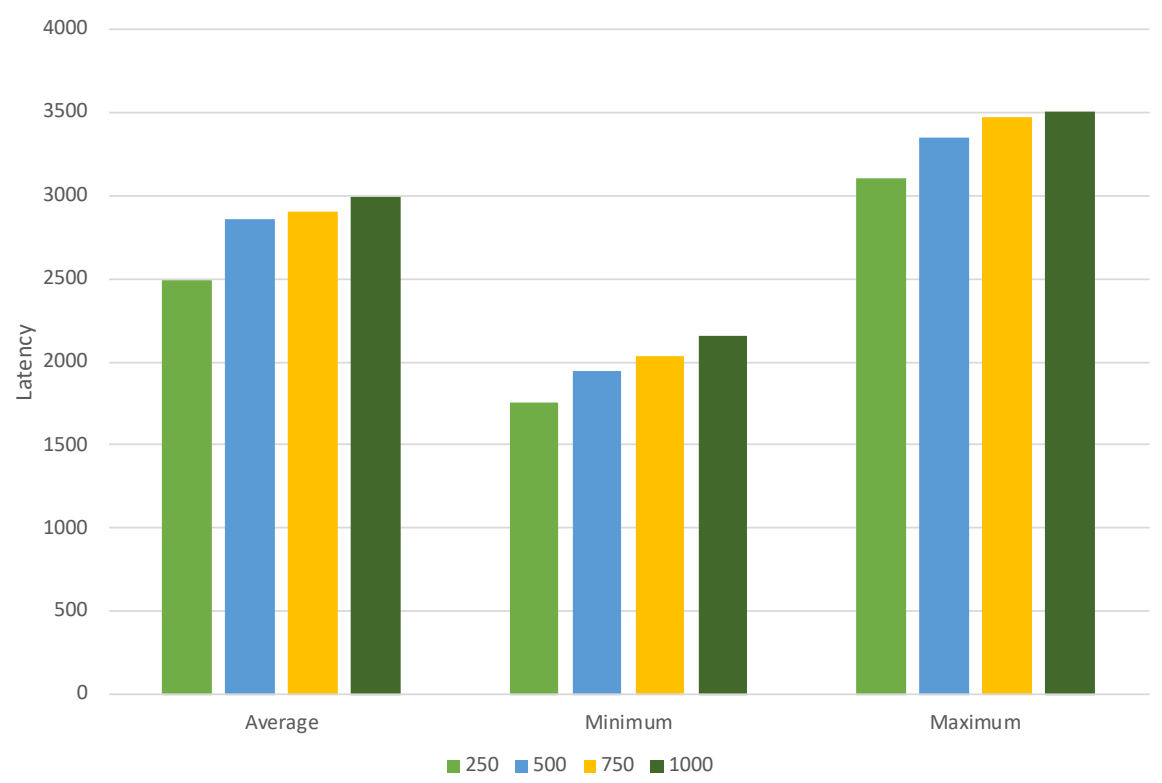

Figure 4: Invoke transaction based latency for different user count

The proposed model is compared and analyzed with the existing state-of-the-art models. This helps in demonstrating the significance and effectiveness of the proposed architecture. The simulation results of both the systems for performance evaluation in terms of processing overhead is provided in Figure 5. Bandwidth, computation time, excess or indirect memory and other resource parameters may be essential for validation of new blocks in blockchain. This is termed as processing overhead. This value is found to be lower in the proposed model when compared with certain existing models. The performance of the proposed system is improved in terms of data security and processing time when compared to the existing models.

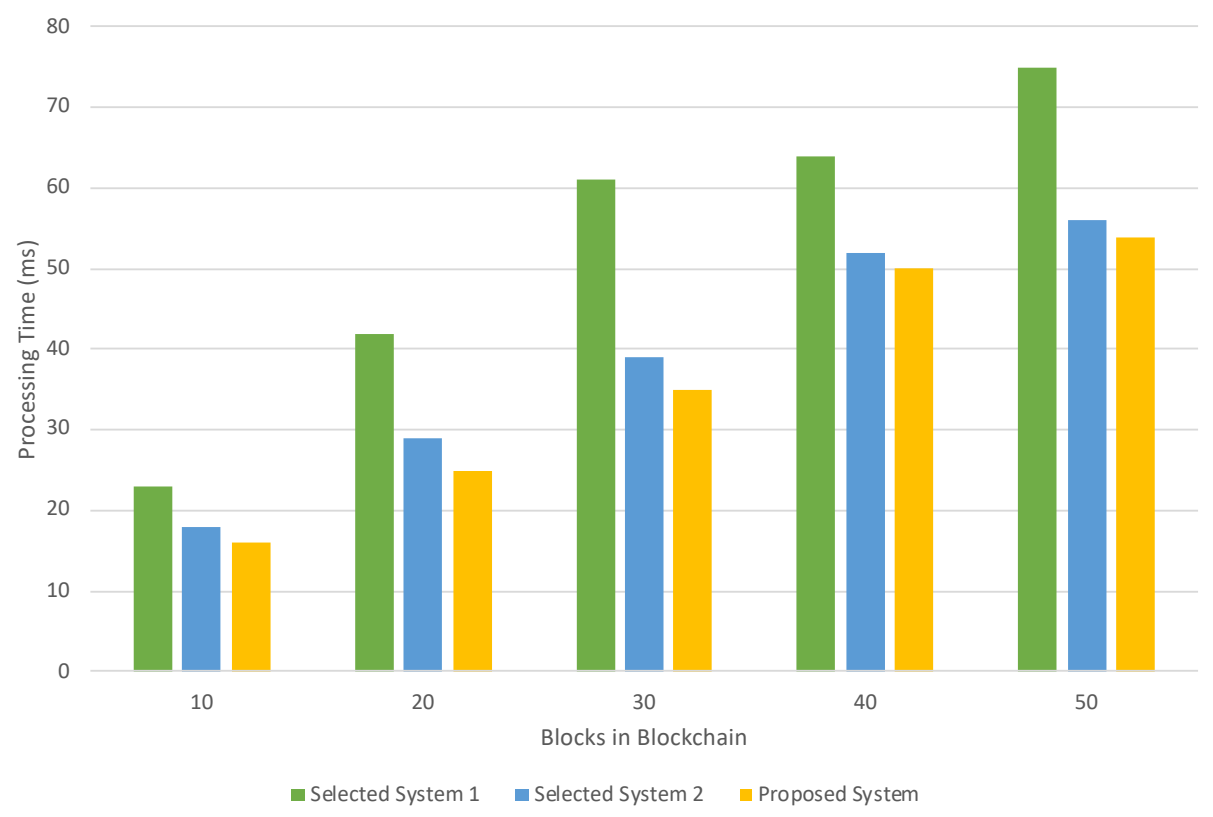

Figure 5: Processing overhead comparison of the proposed and existing IoT healthcare systems 
Journal of ISMAC (2020)

Vol.02/ No.03

Pages: $154-159$

http://irojournals.com/iroismac/

DOI: https://doi.org/10.36548/jismac.2020.3.003

\section{Conclusion}

In this paper, IoT, Blockchain and Cloud technologies are integrated in the medical environment for offering healthcare and tele-medical laboratory services. The vital signs and physiological parameters are sensed and transmitted to provide meaningful, transparent and safe medical assistance to patients. This decentralized platform makes use of Ethereum hybrid network certification system which offers lesser response time and cost when compared to other approaches. The public test instances, robustness and system capabilities are improved along with scalability benefits. Communication is established between the IoT nodes, server and blockchain network in the healthcare domain. The users can interact with the blockchain platform by means of a front-end web application. Various metrices are used for analyzing the performance of the proposed system. Along with the reduction of latency, the overall throughput of the system is increased significantly using this architecture. This system can be tested using various IoT frameworks for its interoperability as a future direction.

\section{References}

[1] Siyal, A. A., Junejo, A. Z., Zawish, M., Ahmed, K., Khalil, A., \& Soursou, G. (2019). Applications of blockchain technology in medicine and healthcare: Challenges and future perspectives. Cryptography, 3(1), 3.

[2] Dwivedi, A. D., Srivastava, G., Dhar, S., \& Singh, R. (2019). A decentralized privacy-preserving healthcare blockchain for IoT. Sensors, 19(2), 326.

[3] Dubovitskaya, A., Xu, Z., Ryu, S., Schumacher, M., \& Wang, F. (2017). Secure and trustable electronic medical records sharing using blockchain. In AMIA annual symposium proceedings (Vol. 2017, p. 650). American Medical Informatics Association.

[4] Kaur, H., Alam, M. A., Jameel, R., Mourya, A. K., \& Chang, V. (2018). A proposed solution and future direction for blockchain-based heterogeneous medicare data in cloud environment. Journal of medical systems, 42(8), 156.

[5] Shae, Z., \& Tsai, J. J. (2017, June). On the design of a blockchain platform for clinical trial and precision medicine. In 2017 IEEE 37th international conference on distributed computing systems (ICDCS) (pp. 19721980). IEEE.

[6] Al Omar, A., Bhuiyan, M. Z. A., Basu, A., Kiyomoto, S., \& Rahman, M. S. (2019). Privacy-friendly platform for healthcare data in cloud based on blockchain environment. Future generation computer systems, 95, 511 521.

[7] Khatoon, A. (2020). A blockchain-based smart contract system for healthcare management. Electronics, 9(1), 94.

[8] Khezr, S., Moniruzzaman, M., Yassine, A., \& Benlamri, R. (2019). Blockchain technology in healthcare: A comprehensive review and directions for future research. Applied sciences, 9(9), 1736.

[9] Choudhury, O., Fairoza, N., Sylla, I., \& Das, A. (2019). A blockchain framework for managing and monitoring data in multi-site clinical trials. arXiv preprint arXiv:1902.03975.

[10] Sivaganesan, D. (2019). DESIGN AND DEVELOPMENT AI-ENABLED EDGE COMPUTING FOR INTELLIGENT-IOT APPLICATIONS. Journal of trends in Computer Science and Smart technology (TCSST), 1(02), 84-94.

[11] Mugunthan, S. R. (2019). Security and Privacy Preserving Of Sensor Data Localization Based On Internet of Things. Journal of ISMAC, 1(02), 81-92.

[12] Haoxiang, W. (2019). Trust management of communication architectures of internet of things. Journal of trends in Computer Science and Smart technology (TCSST), 1(02), 121-130.

[13] Shakya, S. (2019). EFFICIENT SECURITY AND PRIVACY MECHANISM FOR BLOCK CHAIN APPLICATION. Journal of Information Technology, 1(02), 58-67.

[14] Sivaganesan, D. (2019). Block chain enabled internet of things. Journal of Information Technology, 1(01), $1-8$.

[15] Shen, M., Deng, Y., Zhu, L., Du, X., \& Guizani, N. (2019). Privacy-preserving image retrieval for medical IoT systems: A blockchain-based approach. IEEE Network, 33(5), 27-33. 